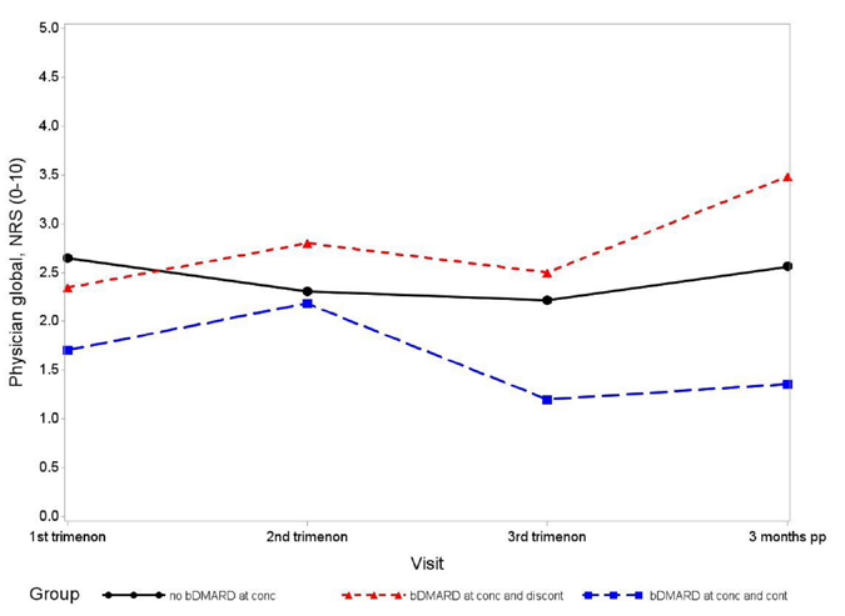

Figure 1 Course of physician assessed global disease activity

Disclosure of Interests: None declared.

DOI: 10.1136/annrheumdis-2021-eular.2087

\section{AB0473 TREATMENT OF ANKYLOSING SPONDYLITIS (AS) DURING PREGNANCY}

O. Krichevskaya ${ }^{1}$, T. Dubinina ${ }^{1}$, E. llinykh ${ }^{1}$, S. Glukhova ${ }^{1}$, A. Demina ${ }^{1}{ }^{1}$ V. A. Nasonova Research Institute of Rheumatology, Laboratory of Medical and Social Problems of Rheumatology, Moscow, Russian Federation

Background: The use of medications during pregnancy remains a challenge for both rheumatologists and patients. The lack of compelling evidence of the safety of a line of different drugs, as well as the traditional idea of the incompatibility of therapy and gestation that is common view in Russia, lead to unjustified withdrawal of drugs by both pregnant women and rheumatologists.

Objectives: to describe the frequency of drug usage in pregnant women with AS, to determine the relationship of AS activity and pregnancy complications.

Methods: 49 pregnant women with confirmed AS (modified New York criteria, 1984) were included for prospective observation. 50 pregnancies in total were traced. The average age of the patients was $31.6 \pm 4.9$ years, the duration of the disease was $134.4 \pm 85.8$ months. The visits were conducted at 10-11, 20-21, and 31-32 weeks of pregnancy. The BASDAl in the month of conception and in the trimesters of pregnancy was: $1,4[0,6 ; 3,3] ; 2,3[1,2 ; 4,4] ; 2,8[1,4$; $4,2]$ and $2,2[1,6 ; 4,0]$, respectively. 48 pregnancies ended with the birth of alive children at an average of $39 \pm 1.1$ weeks, the height of newborns $-51.6 \pm 2.1 \mathrm{~cm}$, weight-3397.3 $\pm 433.1 \mathrm{~g} .8$ (16.3\%) newborns had malformations, 7 of them - minor heart development anomalies, 1 - hydronephrosis. Pregnancy complications: early toxicosis - $18 \%$ of pregnant women, threatening of early abortion $10 \%$, threatening of late abortion- $4.1 \%$, threatening of early preterm birth $-6.3 \%$; premature birth $-3(6.3 \%)$ at $36.8 \pm 0.1$ weeks.

Results: NSAIDs. After inclusion in the study, the drug of choice was ibuprofen, which was canceled for all women no later than the 32-nd week of pregnancy. At the time of conception and in the first, second and third trimesters of pregnancy, NSAIDs were taken by $23(46 \%), 20(40 \%), 30(60 \%)$ and 21 (43.8\%) women, respectively. No effect of NSAIDs on the activity of AS was revealed.

Sulfasalazine (SS) was taken for 3 months before pregnancy by $11(22 \%)$ women, during pregnancy $-6(12 \%)$ at a dose of $1.25 \pm 0.25 \mathrm{~g}$ because of arthritis. The withdrawal of SS was not associated with the recurrence of arthritis.

Glucocorticoids (GC) at a dose of $7.5 \pm 2.5 \mathrm{mg} 3$ months before pregnancy and in the trimesters of pregnancy were taken: $1(2 \%), 4(8 \%), 8(16 \%)$ and $10(20.8 \%)$ women, of whom 1 patient had arthritis and 1 had inflammatory bowel disease. The remaining patients received GC due to the high activity of AS due to axial manifestations and the unavailability of bDMARDs) intake. Against the background of taking GC, the AS activity did not decrease: BASDAl in the 2nd and 3rd trimesters was $5.5 \pm 0.6$ and $5.8 \pm 1.3(p>0.05)$.

bDMARDs. TNF inhibitors for 3 months before pregnancy and on trimesters were received by $11(22 \%), 7(14 \%), 6(12 \%)$ and $2(4.2 \%)$ patients, of which 1 woman in the second trimester obtained firstly initiated therapy with certolizumab pegol due to the recurrence of uveitis. In those who canceled bDMARDs therapy (both independently and due to the recommendation of a rheumatologist), an increase in AS activity was noted on the eve of pregnancy; BASDAI in the month of conception and in the I, II, and III trimesters was: $2,7[0,8 ; 3,5] ; 5,1[3,1 ; 5,9] ; 5,5$ $[5 ; 6]$ and $6,7[5,3 ; 7 ; 3], p<0,05$ compared to the month of conception. Undoing the bDMARDs in the month of conception is a risk factor for high activity as
(BASDAl>4) in the second trimester (OR OF $30.4 ; 95 \% \mathrm{Cl} 1,5-612,3 ; \mathrm{p}=0.03$ ) and in the third trimester (OR A 32.7; 95\% $\mathrm{Cl} 1,6-662,2 ; \mathrm{p}=0.02$ ).

The relationship between pregnancy complications, malformations of the newborns and the used medications was not revealed.

Conclusion: NSAIDs and GC in low doses do not reduce the activity of AS. Withdrawal of TNF inhibitors on the eve of pregnancy is a predictor of high AS activity. It is necessary to increase the knowledge of rheumatologists and patients about the therapeutic possibilities during pregnancy to avoid unjustified drug withdrawal.

Disclosure of Interests: None declared.

DOI: 10.1136/annrheumdis-2021-eular.2427

\section{\begin{tabular}{|l|l}
\hline AB0474 & THE USE OF NONSTEROIDAL ANTI-INFLAMMATORY
\end{tabular} DRUGS (NSAIDS) IN WOMEN WITH ANKYLOSING SPONDYLITIS (AS) DURING PREGNANCY}

O. Krichevskaya ${ }^{1}$, T. Dubinina ${ }^{1}$, E. llinykh ${ }^{1}$, S. Glukhova ${ }^{1}$, A. Demina ${ }^{1} .{ }^{1}$ V. A. Nasonova Research Institute of Rheumatology, Laboratory of Medical and Social Problems of Rheumatology, Moscow, Russian Federation

Background: NSAIDs remain the first-line drugs in treatment of AS. During pregnancy, COX-2 non-selective NSAIDs are allowed for intake up to 32 weeks, but the question of the dose-dependent effect of NSAIDs on fetal organogenesis in the 1st trimester and on fetal kidney function and the increased risk of bleeding in childbirth when taken in the second half of pregnancy continues to be discussed. At the same time, data on the effectiveness of NSAIDs, including their low and medium doses, during pregnancy are extremely small.

Objectives: to describe the frequency of using NSAIDs during pregnancy, to determine relationship between the dose of NSAIDs, adherence to therapy with the activity of AS.

Methods: 50 pregnancies were followed in 49 pregnant women with confirmed AS (modified New York criteria, 1984). The average age of the pts was $31.6 \pm$ 4.9 years, the duration of the disease was $134.4 \pm 85.8$ months. The visits were conducted at 10-11, 20-21, and 31-32 weeks of pregnancy. The BASDAl in the month of conception and in the trimesters (trim.) of pregnancy was: $1,4[0,6$; $3,3] ; 2,3[1,2 ; 4,4] ; 2,8[1,4 ; 4,2]$ and $2,2[1,6 ; 4,0]$, respectively. The level of nocturnal back pain according to the NRS in the first, second and third trim. was: $3.2 \pm 2.0 ; 5.4 \pm 2.5$ and $5.2 \pm 2.6$, respectively. The drug of choice was ibuprofen at a maximum daily dose of $1200 \mathrm{mg}$, its withdrawal - no later than 32 weeks of pregnancy.

Adherence to NSAID therapy was defined as the ratio of the actual dose taken to the prescribed dose; an indicator of less than $80 \%$ was regarded as non-adherence to therapy. The total dose of NSAIDs was determined by the NSAID intake index (M. Dougados, 2001). The" actual daily dose" of ibuprofen was the sum of the doses of ibuprofen taken, divided by the number of actual days of taking the drug. The "average daily dose" was defined as the sum of the ibuprofen doses taken, divided by the number of days in the trimester.

Results: At the time of conception and in the first, second and third trim. of pregnancy, NSAIDs were taken $23(46 \%), 20(40 \%), 30(60 \%)$ and $21(43.8 \%)$ women, respectively. The NSAID intake index, the actual and average daily dose of ibuprofen are shown in the Table 1.

\begin{tabular}{lcccc}
\hline & $\begin{array}{c}\text { month of } \\
\text { conception }\end{array}$ & trim. 1 & trim. 2 & trim. 3 \\
\hline the actual daily dose, $\mathrm{mg}$ & - & 700 & 800 & 750 \\
& & {$[425 ; 800]$} & {$[400 ; 1000]$} & {$[400 ; 1200]$} \\
the average daily dose, $\mathrm{mg}$ & - & 158 & 355,1 & 580 \\
& & {$[87,9 ; 307,7]$} & {$[138,5 ; 685,7]$} & {$[320 ; 1200]$} \\
NSAIDs intake index & 28,6 & 5,8 & 15,5 & 24,4 \\
& {$[16,7 ; 50]$} & {$[2,9 ; 11,8]$} & {$[4,7 ; 30,9]$} & {$[9,5 ; 50]$} \\
\hline
\end{tabular}

The index of NSAID intake in the first trim. was lower than before pregnancy and in the second half of gestation ( $p<0.05$ compared to the month of conception, II and III trim.). The average daily dose of ibuprofen was also lower in the first trim. than in the second and third trim. $(p<0.05)$, while the actual daily dose in the second trim. was higher than in the first and third trim. $(p<0.05$ in all cases).

There was no correlation between BASDAI AS activity, the level of nocturnal pain and the ibuprofen intake index, likewise the fact of NSAID withdrawal throughout pregnancy. In addition, there were no differences in BASDAI levels and back pain in women with a subjective need for NSAIDs, who did and did't take ibuprofen. $50 \%$ of women were committed to NSAID therapy in the first trim., $43.5 \%$ in the second trim., and $67.4 \%$ in the third trim. In pts with non-adherence to NSAID therapy, the BASDAI level was higher than in those who followed the recommendations of the rheumatologist throughout pregnancy: in the first trim. $-3.8[3.4$ 4.7] and $1.7[0.8 ; 2.2]$; in the second trim. - 3[2.3; 4.6] and $1.4[0.8 ; 2.7]$; in the 